\title{
Noise dependence on temperature in fluxgates with electroplated core is
}

\author{
Mattia Butta 워, Michal Přibil, Pavel Ripka
}

円 Show more

https://doi.org/10.1016/j.sna.2016.04.018

Get rights and content

\section{Highlights}

- We tested the noise dependence of fluxgates with electroplated NiFe ring cores.

- The noise shows to be temperature-dependent only in few cases.

- The temperature dependence of the noise is not correlated with the magnetostriction.

- Investigation of the cooling rate of the ring after electroplating shows that thermal shock is a cause of excessive noise in the fluxgate.

\section{Abstract}

Samples of electrodeposited fluxgate sensors with varying magnetostriction coefficient were manufactured and their noise was studied at various temperatures. We show that the noise of the fluxgate is, under some conditions, dependent on temperature. We studied such behaviour of the noise for samples with low and high magnetostriction and we verified that the both types of sensors presented low and high temperature dependent noise. Then we investigated as a possible source of noise the thermal cooling rate applied to the samples after electroplating and we verified that large thermal shock could induce mechanical stress and noise in the sensor.

$<$ Previous

Next $>$

\section{Keywords}

Fluxgate; Noise; Magnetostriction; Electrodeposition; NiFe

\section{Introduction}

Fluxgate sensors are often used to measure magnetic field at low frequency in environments where the temperature cannot be controlled. Offset stability of fluxgate has been extensively studied together with the effect of magnetostriction on it in Ref. [1]. However, the dependence of noise on temperature was measured only on amorphous [2], [3] or nanocrystalline [4] cores. We have already shown that for electrodeposited crystalline cores the minimum noise is reached for zero-magnetostriction composition [5]. In this paper we study the temperature effect using six-layer magnetic shielding with internal thermostat. Our shielding has Dewar flask to isolate temperature-controlled environment from the magnetic shielding material so it has constant temperature and the measured noise is not affected by the change of shielding remanence. We acquired noise spectra in a $0.2 \mathrm{~Hz}-200 \mathrm{~Hz}$ band (lower frequency could not be measured due to temperature changes in time). 
The cores have been electroplated using a classical Watts-type bath at $55^{\circ} \mathrm{C}$ on $0.25 \mathrm{~mm}$ thick fiberglass ring covered by $9 \mu \mathrm{m}$ copper layer (Fig. 1). The rings had $44 \mathrm{~mm}$ inner diameter and $48 \mathrm{~mm}$ external diameter. In order to produce samples with different magnetostriction we changed the current density of electroplating, which in turn modifies the composition of the electroplated film. Samples B01, B02 and B03 were electrodeposited using $J=16 \mathrm{~mA} / \mathrm{cm}^{2}$ current density, which should give a minimum magnetostriction (81Ni19Fe composition). Samples BP1 and BP2 were electrodeposited using $32 \mathrm{~mA} / \mathrm{cm}^{2}$ and $48 \mathrm{~mA} / \mathrm{cm}^{2}$ current density, respectively, yielding higher iron content in the composition and thus positive coefficient of magnetostriction. Sample BN1, on the other hand, was electrodeposited with only $11.2 \mathrm{~mA} / \mathrm{cm}^{2}$, which causes higher content of nickel and, consequently, negative coefficient of magnetostriction [5]. Concrete values of magnetostriction are presented in Table 1. While sample B01 shows indeed low magnetostriction as expected, samples B02 and B03 show slightly larger magnetostriction, but in any case it is still lower than the magnetostriction obtained with larger or lower current density. The electroplating time at $J=16 \mathrm{~mA} / \mathrm{cm}^{2}$ is $30 \mathrm{~min}$, whereas for the other samples we changed the deposition time in inverse proportion to the current density to obtain a uniform thickness (estimated to be about $6 \mu \mathrm{m}$ ) in all samples independently on the current density. In Table 1 we included also the estimated composition of the rings derived by the electroplating current, based on the dependence of composition on electroplating current presented in Ref. [5].

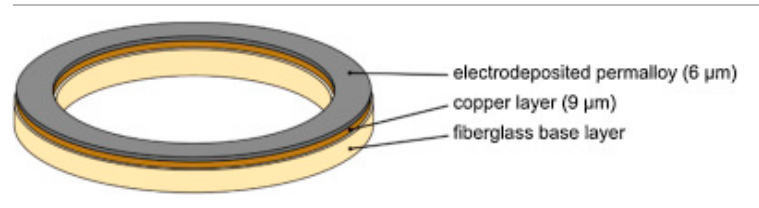

Download high-res image (114KB)

Download full-size image

Fig. 1. Structure of the electrodeposited cores (dimensions exaggerated for illustration).

Table 1. Coefficients of magnetostriction of measured samples.

\begin{tabular}{llll}
\hline & $\mathrm{J}\left(\mathrm{mA} / \mathrm{cm}^{2}\right)$ & Magnetostriction $(-)$ & Composition [\%Fe-\%Ni] \\
\hline BN1 & 11.2 & $-2.68 \times 10^{-5}$ & $12-88$ \\
B01 & 16 & $0.63 \times 10^{-5}$ & $19-81$ \\
B02 & 16 & $1.4 \times 10^{-5}$ & $19-81$ \\
B03 & 16 & $2.4 \times 10^{-5}$ & $19-81$ \\
BP1 & 32 & $3 \times 10^{-5}$ & $22-78$ \\
BP2 & 48 & $4.2 \times 10^{-5}$ & $32-68$ \\
\hline
\end{tabular}

\section{Measurement setup}

The rings have then been used as core for fluxgate with excitation winding with 540 turns and $2.6 \mathrm{~A}_{\mathrm{p}-\mathrm{p}}$ excitation current at $30 \mathrm{kHz}$ frequency. The pick-up coil had 450 turns and it was tuned by external capacitor (which was kept at room temperature). The second harmonic of the voltage induced in the pick-up coil was extracted by means of a DSP lock-in amplifier. The sensor was placed in a thermally regulated shielding (Fig. 2) and its noise recorded in a $0.2 \mathrm{~Hz}-200 \mathrm{~Hz}$ frequency range with 50 averaging (no overlapping). The temperature in the shielding was changing at most $\pm 0.5^{\circ} \mathrm{C}$ during the whole process of spectra measurement and averaging, so that the noise spectra measured was not influenced by the change of temperature, which we can consider constant from this point of view. 


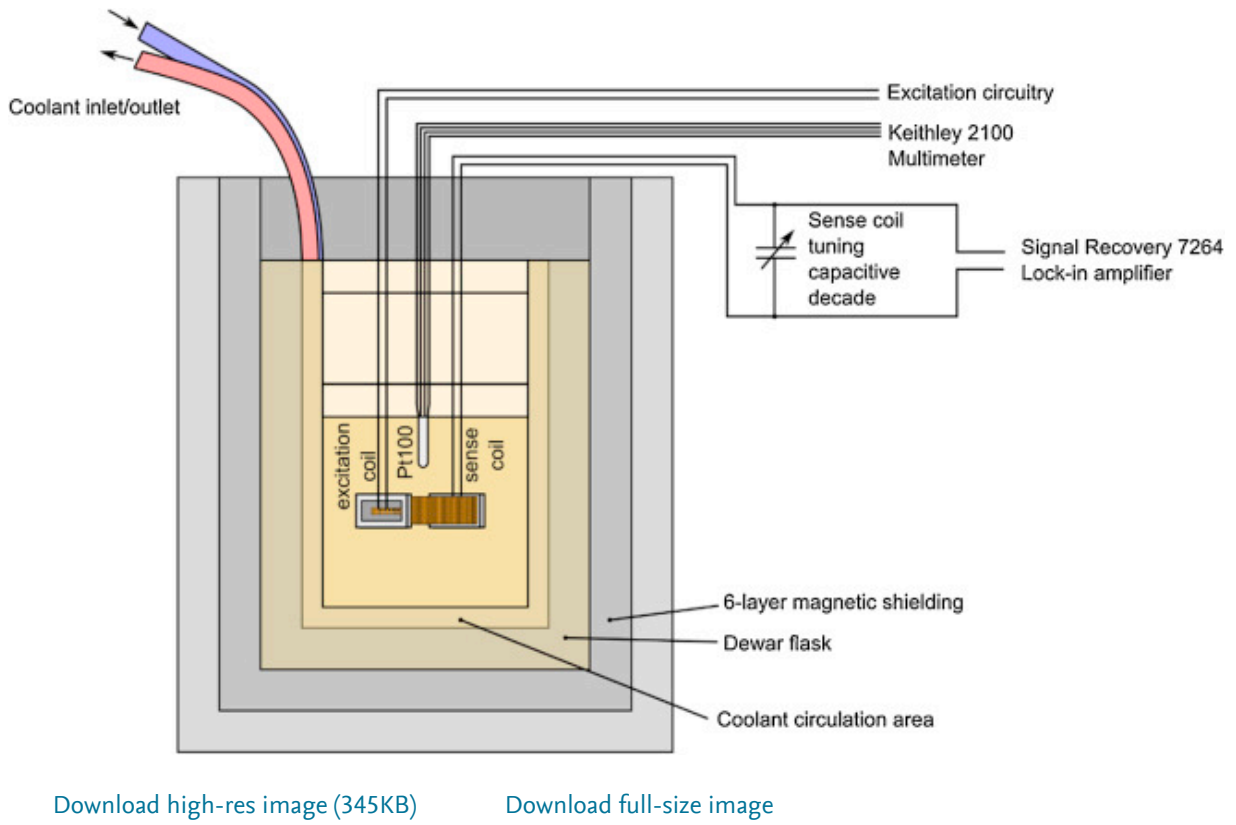

Fig. 2. Simplified schematic drawing of thermostatic shielding configuration.

\section{Results}

The dependence of noise at $1 \mathrm{~Hz}$ and $10 \mathrm{~Hz}$ of all the electrodeposited sensors is shown in Fig. 3, Fig. 4, respectively. As expected the cores electroplated with $J=16 \mathrm{~mA} / \mathrm{cm}^{2}$ show lower noise than the cores electroplated with higher and lower current density, due to the magnetostriction. However, we observe that for some sensors, the noise is independent of temperature, while in others, the noise decreases with growing temperature. It is important to point out that the slope of temperature dependence in the latter is very similar in logarithmic scale, and therefore, the relative change in the noise is also similar.

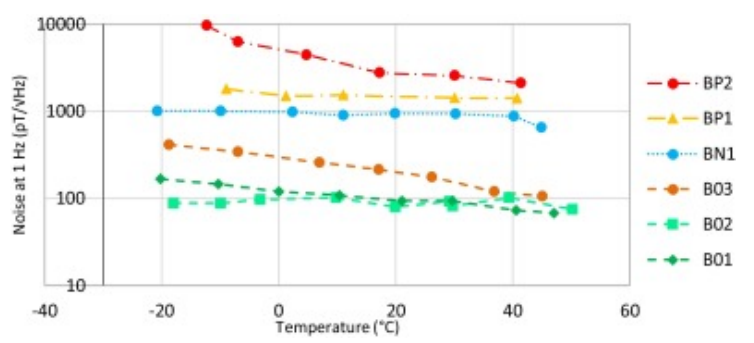

Download high-res image (122KB) Download full-size image

Fig. 3. Noise amplitude spectrum densities of samples with varying magnetostriction at $1 \mathrm{~Hz}$ versus temperature.

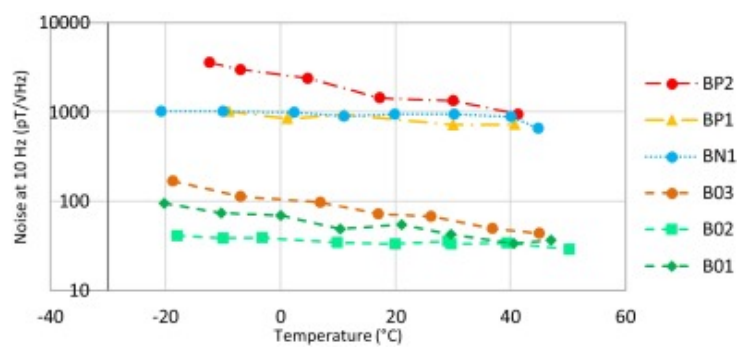

Download high-res image (121KB) Download full-size image

Fig. 4. Noise amplitude spectrum densities of samples with varying magnetostriction at $10 \mathrm{~Hz}$ versus temperature.

Additionally we can see in Fig. 5 the power spectral density of the noise of two samples, with low magnetostriction (left) and with high magnetostriction (right) for three values of temperature ranging form $-20^{\circ} \mathrm{C}$ to $+47^{\circ} \mathrm{C}$. We can see that when the noise changes with temperature the spectrum is almost uniformly decreasing with increasing noise for all the considered frequency range. This assures the

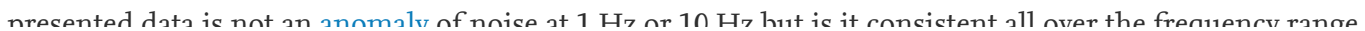



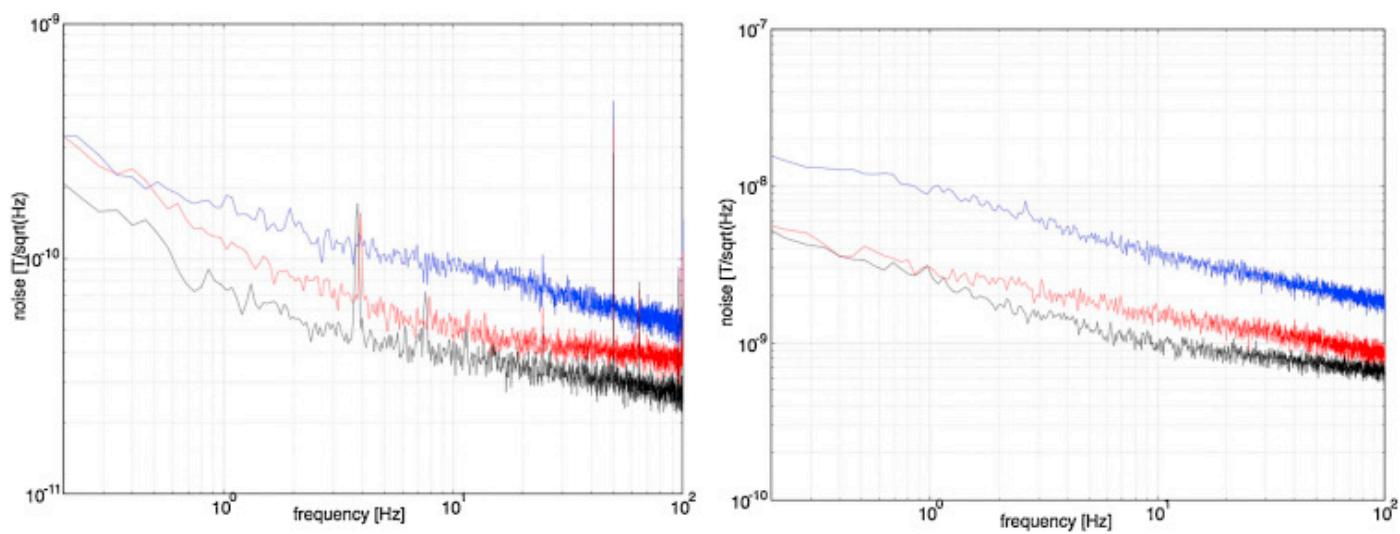

Download high-res image $(618 \mathrm{~KB})$

Download full-size image

Fig. 5. Left: power spectral density of sample with low magnetostriction (left) measure at $-20.3^{\circ} \mathrm{C}$ (blue), $+21^{\circ} \mathrm{C}$ (red) and $+47^{\circ} \mathrm{C}$ (black). Right: power spectral density of sample with high magnetostriction at $-12^{\circ} \mathrm{C}$ (blue) $+17^{\circ} \mathrm{C}$ (red) and $+41^{\circ} \mathrm{C}$ (black). (For interpretation of the references to color in this figure legend, the reader is referred to the web version of this article.)

Assuming that magnetostriction is the dominant cause of the noise in the presented sensors, this indicates it is the magnetostriction noise itself what is temperature dependent. The cause of this unusual behaviour in supposedly identical sensors (B01, B02 and B03) was unclear. As the electroplating was made at $55^{\circ} \mathrm{C}$ we expect that the rings might have mechanical stress at temperature lower than $55^{\circ} \mathrm{C}$ and this stress is reduced as the temperature increases and approaches the electroplating temperature. However, this phenomenon is observed only for some sensors. A possible explanation can be found in the cooling process of the cores after electroplating, because in some cases the cores were washed in running water (bringing to high rate cooling) whereas in others the cores were cooled down naturally at room temperature without thermal shock. This could give rise to mechanical stress in the electroplated film, which eventually vanishes as the temperature approaches the temperature the sample was electroplated at. This would also explain the scatter of noise observed at room temperature for sensors electroplated apparently under the same conditions (except for the cooling process). Unfortunately, this was not considered before the process of electro deposition and, therefore, the post-treatment of the cores was not documented. In order to validate this hypothesis we performed the experiment described in the following paragraph.

We have performed an additional test to verify that the change of noise vs. temperature observed for some sensors was no given by the change of sensitivity of the sensor itself. Unfortunately we could not directly measure the sensitivity for different temperature because we cannot generate a test field inside the shielding with thermostatically adjusted temperature without magnetizing the shielding itself. Therefore, we verified the sensitivity indirectly. We measured the series inductance of the magnetic cores used for this study in a range of temperature from about $-10{ }^{\circ} \mathrm{C}$ to $50{ }^{\circ} \mathrm{C}$. The dependence of the inductance of each core, normalized to the value of the inductance at $18{ }^{\circ} \mathrm{C}$ is shown in Fig. 6 . We can see that there is a slight change of inductance (and therefore sensitivity) for all cores, but in the worst case this is at most $\pm 7 \%$ of the value at $50{ }^{\circ} \mathrm{C}$. Such variation are totally uncorrelated to the variation of noise we observed in Fig. 3, Fig. 4. This means that any variation of noise vs. temperature could not be caused by such minor change of sensitivity.

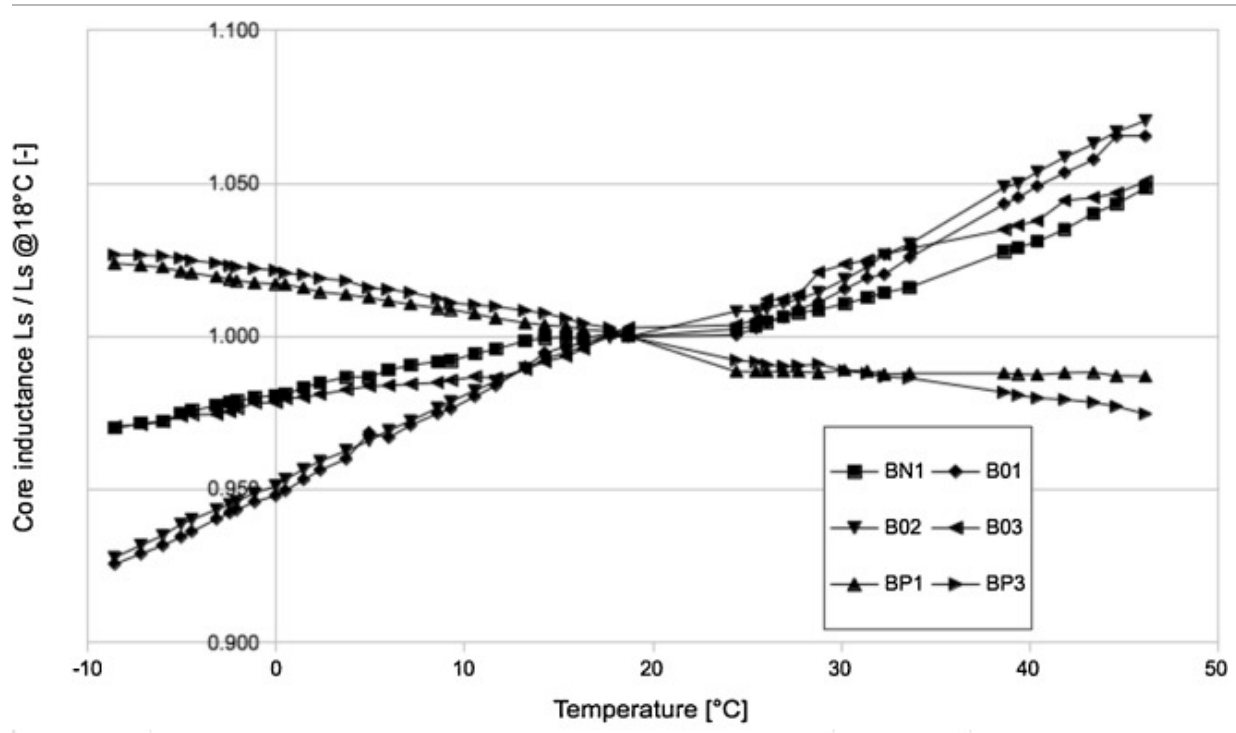

Download high-res image (298KB) Download full-size image

Fig. 6. Dependence of the series inductance (divided by its value at $18^{\circ} \mathrm{C}$ ) vs. temperature. 


\section{Effect of cooling rate after electroplating}

We manufactured twelve rings using under the same conditions. The current density was $16 \mathrm{~mA} / \mathrm{cm}^{2}$ in all cases to assure a low magnetostriction for all rings. The electroplating time was $1 \mathrm{~h}$ for all samples and also the temperature of electroplating was $55^{\circ} \mathrm{C}$ in all baths. However, six rings were exposed to a slow natural cooling after electroplating from $55{ }^{\circ} \mathrm{C}$ to room temperature, whereas the remaining six rings were forced to a fast cooling by dropping them in cold water (about $2{ }^{\circ} \mathrm{C}$ ) right at the end of the electroplating process. Each bath was used only for two rings to assure the composition of the bath was uniform for all rings. Our idea was to induce a thermal shock to intentionally produce stress between the magnetic layer and the substrate it was electroplated on. In this way we can verify if it is true that some additional noise in the sensor is due to the thermal shock after electroplating. The rings have been tested as cores for fluxgate as previously described. Fig. 7 shows the power spectral density of the noise at $1 \mathrm{~Hz}$ for all twelve rings.

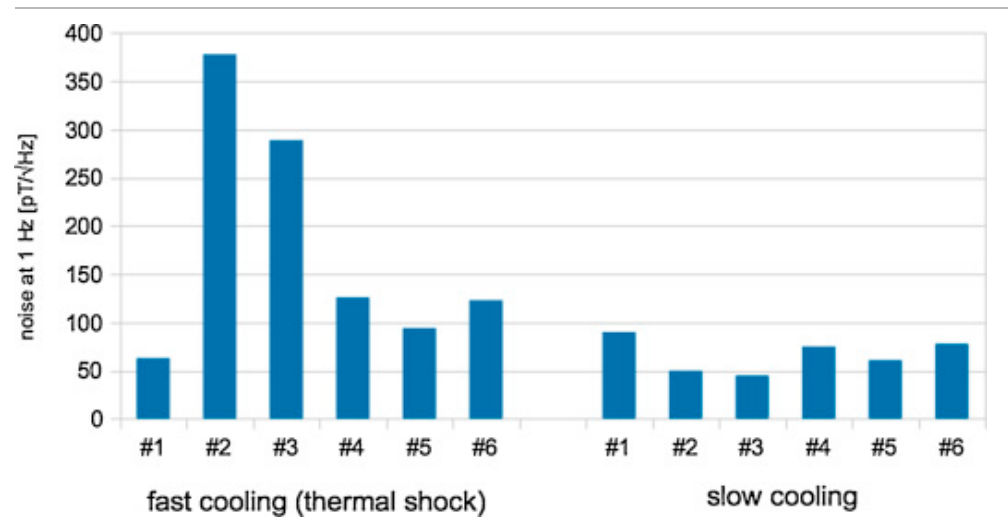

Download high-res image (193KB) Download full-size image

Fig. 7. Power spectral density of the noise at $1 \mathrm{~Hz}$ for fluxgates based on electrodeposited rings exposed to fast or slow cooling after electroplating.

As usual there is some scatter of noise from sample to sample, however we can notice the sensors based on rings exposed to thermal shock have a larger noise than the rings which underwent a slow cooling. Even if the difference is not extremely large the effect of the thermal shock looks clear. This result suggests that in the manufacturing process of electroplated rings special care should always be paid to assure a slow cooling (interestingly this issue has never been documented in literature). Moreover, this experiments highlight the need for a new type of substrate for electroplated rings. If noise has to be reduced, material with temperature dilatation coefficient similar to Permalloy has to be used as substrate for the electroplated rings.

\section{Conclusion}

We examined temperature dependence of the noise of fluxgate sensors with electrodeposited permalloy cores. The main finding is that slow cooling after electrodeposition is important. The achieved noise level of $50 \mathrm{pT} / \mathrm{V} \mathrm{Hz}$ is comparable to noise of pcb fluxgates with amorphous cores [6], while electrodeposited permalloy as a material for fluxgate sensor core is more convenient for the mass production [7].

\section{Acknowledgment}

This work has been supported by the Grant Agency of the Czech Republic (GACR) under the grant P102/12/2177.

Recommended articles Citing articles (0)

\section{References}

[1] P. Ripka, M. Pribil, M. Butta

Magnetostriction offset of fluxgate sensors

IEEE Trans. Magn., 51 (2015)

(Article\#: 4000504)

Google Scholar

[2] B.B. Narod, J.R. Bennest, J.O. Stromolsen, et al.

An evaluation of the noise performance of $\mathrm{Fe}, \mathrm{Co}$, $\mathrm{Si}$, and $\mathrm{B}$ amorphous-alloys in ring-core fluxgate magnetometers

Can. J. Phys., 63 (1985), pp. 1468-1472

CrossRef View Record in Scopus Google Scholar

[3] Y. Nishio, F. Tohyama, N. Onishi

The sensor temperature characteristics of a fluxgate magnetometer by a wide-range temperature test for a mercury exploration satellite 
Meas. Sci. Technol., 18 (2007), pp. 2721-2730

CrossRef View Record in Scopus Google Scholar

[4] D. Rühmer, S. Bögeholz, F. Ludwig, M. Schilling

Vector fluxgate magnetometer for high operation temperatures up to $250^{\circ} \mathrm{C}$

Sens. Actuator A-Phys., 228 (2015), pp. 118-124

Article Download PDF View Record in Scopus Google Scholar

[5] M. Butta, M. Janosek, P. Ripka, L. Kraus, R. Kammouni

Influence of magnetostriction of NiFe electroplated film on the noise of fluxgate

IEEE Trans. Magn., 50 (2014)

(Article\#: 4006504)

Google Scholar

[6] C.-C. Lu, J. Huang, P.-K. Chiu, S.-L. Chiu, J.-T. Jeng

High-sensitivity low-noise miniature fluxgate magnetometers using a flip chip conceptual design

Sens. (Basel Switz.), 14 (8) (2014), pp. 13815-13829, 10.3390/s140813815

CrossRef View Record in Scopus Google Scholar

[7] Jian Lei, Chong Lei, Yong Zhou

Analysis and comparison of the performance of MEMS fluxgate sensors with permalloy magnetic cores of different structures Measurement, 46 (1) (2013)

Google Scholar

Mattia Butta was born in Lecco (Lombardy) in 1980. He received a M.Sc. in Electrical Engineering from Politecnico di Milano in 2005 and a Ph.D. degree from Czech Tecnical University in Prague in 2010. From 2010 and 2012 he was JSPS fellow at Kyushu University. From 2012 he is a researcher at the Magnetic laboratory of department of measurement at FEE-CTU in Prague.

Michal Pribil received a B.sc. And M.Sc. at Faculty of Electrical Engineering, Czech Technical University in 2011 and 2013 respectively. He is currently a Ph.D. student at the same institution.

Pavel Ripka was born in Praha in 1959. He received an Ing. degree in 1984, and CSc. (equivalent to Ph.D.) in 1989, and in Assoc. Prof. degree in 1996. He works at the Department of Measurement, Faculty of Electrical Engineering, Czech Technical University as Prof., lecturing in Measurements, Engineering Magnetism and Sensors. His main research interests are Magnetic Measurements and Magnetic Sensors, especially Fluxgate. He is a co-author of 3 books and > 50 journal papers.

Selected paper from EUROSENSORS 2015 conference, September 6-9, 2015, Freiburg, Germany.

View Abstract

(C) 2016 Elsevier B.V. All rights reserved.

ELSEVIER About ScienceDirect Remote access Shopping cart Advertise Contact and support Terms and conditions Privacy policy

We use cookies to help provide and enhance our service and tailor content and ads. By continuing you agree to the use of cookies. Copyright (C) 2019 Elsevier B.V. or its licensors or contributors. ScienceDirect $₫$ is a registered trademark of Elsevier B.V. 